\title{
A Logic Object and its State
}

\author{
J. Bokr, V. Jáneš
}

This paper shows not only that the state of an objecty parameterizes the dyad (stimulus, response) but that it is also the performer of the state transition initiated by the stimulus. Thus, the logic object is a feedback composition of the logic pseudoobject and the static logic object. It is also stated that, without considering the new role of the state, the content of the concept ,state "cannot be completely explained. The current intuitive conception of canonical decomposition of an entity is confronted with the exactly introduced decomposition and with the mentioned division of the logic object into a pseudoobject and a static object.

Keywords: logic object, pseudoobject, division, canonic decomposition, delay, state.

\section{Introduction}

A real logic object depends on its model, since a model defines an entity. The definition of an object can be understood as a statement of all of its relevant, adequate, and well distinguishable characteristics that factor out the entity from its environment, or document its entirety.

Let us show, on an example of driving a train, the untenable of the current conception of the dynamic logic object.

Example 1: Let the transition table, Tab. 1a), be a model of a train going from point 1 through the position 2 to point 3 . Then the train will go back from position 3 through the point 4 to point 5 . The state of the train is its bold printed position on the track and the actor - the motor - is either at rest or it operates going forward or backward. The ordered pairs of values: 00 and 10 , or 01 of the train control $\mathrm{u}_{1} \mathrm{u}_{2}$ make the train be at rest or go forward or backward, respectively. The flow chart of the conceptions Mealy nondeterministic control automaton of the given train is presented in Tab. 1b) and its minimal form with obvious combinational behavior [2] is shown in Tab. 1c). The control automaton is nondeterministic since if it assumes state 3 of the train, it produces either control 10, and the train remains in state 3 , or 01 , due to which the train goes back to state 5 .
The common statement that control $\mathrm{u}_{1} \mathrm{u}_{2}$ causes the train move from one place to another cannot be accepted, the train not being given data about its position on the track, i.e., without respect to the potential operation of the motor will not move. Thus, control $u_{1} u_{2}$ changing the train's position only initiates, whereas the actor of the train is the starting position of the change of place. Since the transition table Tab. 1a) of the train assumes automatic movement of the train along the track $\rightarrow 1 \rightarrow 2 \rightarrow 3 \rightarrow$ and $\rightarrow 3 \rightarrow 4 \rightarrow 5 \rightarrow$ only due to the respective control 10 or 01 , the model of the train according to Tab. 1a) is inadequate. Moreover, can a nondeterministic control automaton be constructed when an arbitrary product is either nondeterministic, or, if there is no other choice, a pseudodeterministic automation?

In other words, a train modeled by the transition table Tab. 1a) in a finite-semiautomatic way is not (!) a logic object and is referred too only as a logic pseudoobject.

Since the performer of a state transition on a dynamic entity is the starting state of the transition (the transition control is its actor only), we will introduce a logic potentially dynamical pseudoobject [3-5] as a division component (not a decomposition component [4]) of the entity and compare the division of the logic object with its canonic decomposition (not with a structural interpretation of the transition relation,

Table 1: a) Transition table of the train, b) table of the conceptional control automaton, c) table of the minimal control automaton of the train from example 1

\begin{tabular}{|c|c|c|c|}
\hline a) & \multicolumn{3}{|c|}{$s^{\mu+1}$} \\
\hline$u_{s^{\mu}}^{\nu} u_{2}^{v}$ & 00 & 10 & 01 \\
\hline$\rightarrow \quad 1$ & (1) & 2 & \\
\hline 2 & (2) & 3 & \\
\hline 3 & (3) & (3) & 4 \\
\hline 4 & (4) & & 5 \\
\hline 5 & (5) & & (5) \\
\hline
\end{tabular}

\begin{tabular}{|c|c|c|c|c|c|}
\hline b) & \multicolumn{5}{|c|}{$q^{v} / u_{1}^{v} u_{2}^{v}$} \\
\hline$s^{\mu}$ & 1 & 2 & 3 & 4 & 5 \\
\hline$\rightarrow 1$ & $2 / 10$ & & & & \\
\hline 2 & (2) $/ 10$ & $3 / 10$ & & & \\
\hline 3 & & (3)/10 & $4 / 10$ & & \\
\hline 4 & & & (4), $5 / 10,01$ & & \\
\hline 5 & & & (5)/01 & $6 / 10$ & \\
\hline 6 & & & & (6) $/ 01$ & $7 / 01$ \\
\hline 7 & & & & & (7)/01 \\
\hline
\end{tabular}

c)

\begin{tabular}{|l|c|c|c|c|c|}
\hline $\mathrm{s}^{\mu}$ & 1 & 2 & 3 & 4 & 5 \\
\hline $\mathrm{u}_{1} \mathrm{u}_{2}$ & 10 & 10 & 10,01 & 01 & 01 \\
\hline
\end{tabular}


of the function of the object). We will attempt, even if it may be without success, to define the content of the concept of an "object state".

\section{Logic object}

Let the symbol $\mathrm{M}^{\mu}$ denote the set $\{\{\mu\} \rightarrow \mathrm{M}\}$ of all representations $\{\mu\} \rightarrow \mathrm{M}: \mu \longmapsto \mathrm{m}\left(=\mathrm{m}^{\mu}\right)$ and let proj $_{\mathrm{i}}$ be the projection to the i-th axis.

Let the finite-automaton model of the logic pseudoobject $\mathrm{P}$ be an ordered quintet

$$
\mathrm{P}=\left\langle\mathrm{U} \times \mathrm{Z}^{\varepsilon}, \mathrm{S}, \mathrm{Y}, \delta, \lambda\right\rangle \text {. }
$$

Where U, Z, S and Y are the corresponding input alphabet (control alphabet), explicit failure alphabet (if failures occur, then $\varepsilon=1$, otherwise $\varepsilon=0$ ), state and output alphabets, $\delta$ and $\lambda$ is the respective transition relation, or function:

i) $\delta \subseteq S^{\{\mu\}} \times U^{\{v\}} \times S^{\{\mu+1\}}: \delta\left(s^{\mu}, u^{v}, s^{\mu+1}\right)$,

spec. $\delta: S^{\{\mu\}} \times U^{\{v\}} \rightarrow S^{\{\mu+1\}}:\left\langle s^{\mu}, u^{v}\right\rangle \mapsto s^{\mu+1}$

for $\varepsilon=0$

ii) $\delta: S^{\{\mu\}} \times U^{\{v\}} \times Z^{\{\mu\}} \rightarrow S^{\{\mu+1\}}:\left\langle s^{\mu}, u^{v}, z^{\mu}\right\rangle \mapsto s^{\mu+1}$

for $\varepsilon=1$

and the injective Moore output function

$$
\lambda:: \mathrm{S}^{\{\mu\}} \rightarrow \mathrm{Y}^{\{\mu\}}: \mathrm{s}^{\mu} \mapsto \mathrm{y}^{\mu},
$$

when $\mu \in \mathbf{N}_{0}, v \in \mathbf{N}$ (A is a set of natural numbers denoting moments, $\left.\mathbf{N}_{0}=\mathbf{N} \cup\{0\}\right)$. If then $<\left(<\subseteq \mathbf{N}_{0}^{2}\right)$ is a binary relation "before then or simultaneously with" and if by the measuring of $m$ moments the homorphism $\mathfrak{m}: \mathbf{N}_{0} \rightarrow \mathbf{R}_{0}^{+}$is understood, $\left(\mathrm{R}_{0}^{+}\right.$being a set of real nonnegative numbers) such that if $\mu>v$, then $\mathfrak{m}(\mu) \leq \mathfrak{m}(v)$, and afterwards, if $v-1<\mu<v$, then also $\mathfrak{m}(v-1) \leq \mathfrak{m}(\mu) \leq \mathfrak{m}(v)$, see Fig. 1 .

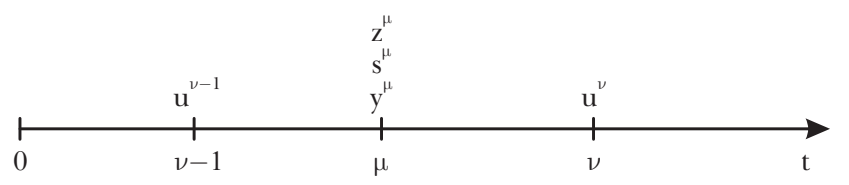

Fig. 1: Time diagram of action $\mathrm{P}$

According to demonstration example 1, we consider the complex of the automatic logic control from Fig. 2 to be incorrect and are no longer concerned with it.

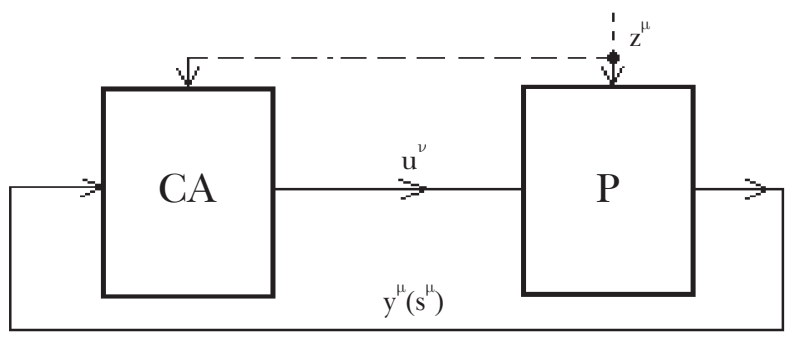

Fig. 2: Complex of automatic logic control of pseudoobject P by a nondeterministic control automaton CA

We require the dynamic logic automatically to have done processes selected by the subject (state trajectories). If the state trajectory contains cycles, we require a finite number of its iterations. Selection of processes in the entity is to be carried ont by the subject by assigning stimuli to the object. A potential carrier of processes on the entity is, without doubt, the given pseudoobject (being unable to perform transitions between the states of the selected state trajectory by itself), since the control of the potentially dynamical pseudoobject only produces the transitions on the pseudoobject. Exaggerating a little, we can refer to the pseudoobject potentially both as "dead" and as a "live". Since the performer of the transition between the pseudoobject states is always the starting state of the state transition, the requirement of automatic operation of the selected process on the object reglements the division of the dynamic object into dividends, which are:

- the given potentially dynamic pseudoobject,

- $\quad$ the selected static logic entity

according to Fig. 3. While at least one part (a dividend) of the object division is a pseudoobject, all parts (components) of the object decomposition are again objects.

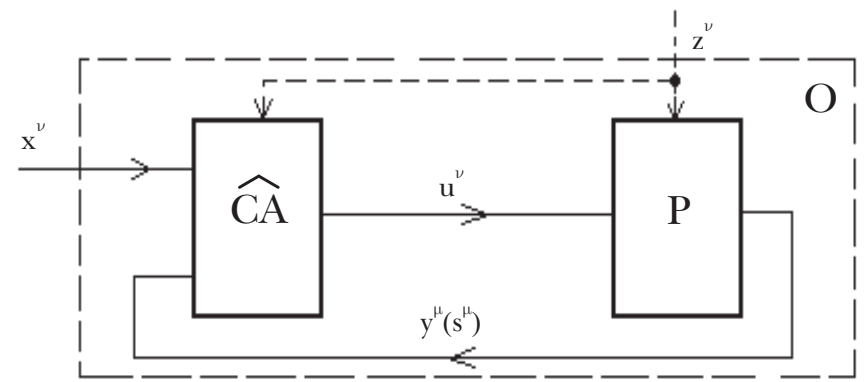

Fig. 3: Division of a dynamic logic object $\mathbf{O}$ into potentially dynamic pseudoobject $\mathbf{P}$ and static logic object $\widehat{\mathrm{CA}}$

Let us prove that the static Mealy pseudoobject

$$
\widehat{\mathrm{CA}}=\left\langle\mathrm{X} \times \mathrm{Y} \times \mathrm{Z}^{\varepsilon}, \mathrm{Q}, \hat{\delta}_{\mathrm{R}}, \hat{\lambda}_{\mathrm{R}}\right\rangle
$$

is a logic object, where $\mathrm{X}$ and $\mathrm{Q}$ are the respective selection alphabet (by selecting a letter from $\mathrm{X}$ the given process on $\mathrm{P}$ can be implemented) and state alphabet, $\hat{\delta}_{\mathrm{R}}$ and $\hat{\lambda}_{\mathrm{R}}$ are the respective transition function:

i) $\hat{\delta}_{\mathrm{R}}: \mathrm{Q}^{\{v-1\}_{\times}} \mathrm{X}^{\{v\}} \times \mathrm{Y}^{\{\mu\}} \rightarrow \mathrm{Q}^{\{v\}}:\left\langle\mathrm{q}^{v-1}, \mathrm{x}^{v}, \mathrm{y}^{\mu}\right\rangle \mapsto \mathrm{q}^{v}$ for $\varepsilon=0$,

ii) $\hat{\delta}_{\mathrm{R}}: \mathrm{Q}^{\{v-1\}} \times \mathrm{X}^{\{v\}} \times \mathrm{Y}^{\{\mu\}} \times \mathrm{Z}^{\{\mu\}} \rightarrow \mathrm{Q}^{\{v\}}:\left\langle\mathrm{q}^{v-1}, \mathrm{x}^{v}, \mathrm{y}^{\mu}, \mathrm{z}^{\mu}\right\rangle \mapsto \mathrm{q}^{v} \quad$ for $\varepsilon=1$

and the Mealy output function:

i) $\hat{\lambda}_{\mathrm{R}}: \mathrm{Q}^{\{v-1\}_{\times}} \mathrm{X}^{\{v\}} \times \mathrm{Y}^{\{v\}} \rightarrow \mathrm{U}^{\{v\}}:\left\langle\mathrm{q}^{v-1}, \mathrm{x}^{v}, \mathrm{y}^{\mu}\right\rangle \mapsto \mathrm{u}^{v} \quad$ for $\varepsilon=0$,

ii) $\hat{\lambda}_{\mathrm{R}}: \mathrm{Q}^{\{v-1\}} \times \mathrm{X}^{\{v\}} \times \mathrm{Y}^{\{v\}} \times \mathrm{Z}^{\{\mu\}} \rightarrow \mathrm{U}^{\{v\}}:\left\langle\mathrm{q}^{v-1}, \mathrm{x}^{v}, \mathrm{y}^{\mu}, \mathrm{z}^{\mu}\right\rangle \mapsto \mathrm{u}^{v} \quad$ for $\varepsilon=1$ 
is a logic object and really, if $Q=\{q\}(|Q|=1)$, we will obtain either:

i) $\hat{\delta}_{\mathrm{R}}:\{\mathrm{q}\} \times \mathrm{X}^{\{v\}} \times \mathrm{Y}^{\{v\}} \rightarrow\{\mathrm{q}\}:\left\langle\mathrm{q}, \mathrm{x}^{v}, \mathrm{y}^{\mu}\right\rangle \mapsto \mathrm{q} \quad$ for $\varepsilon=0$,

ii) $\hat{\delta}_{\mathrm{R}}:\{\mathrm{q}\} \times \mathrm{X}^{\{v\}} \times \mathrm{Y}^{\{v\}} \times \mathrm{Z}^{\{\mu\}} \rightarrow\{\mathrm{q}\}:\left\langle\mathrm{q}, \mathrm{x}^{v}, \mathrm{y}^{\mu}, \mathrm{z}^{\mu}\right\rangle \mapsto \mathrm{q} \quad$ for $\varepsilon=1$,

which can be formally (not actually) ignored, or:

i) $\hat{\lambda}_{\mathrm{R}}:\{\mathrm{q}\} \times \mathrm{X}^{\{v\}} \times \mathrm{Y}^{\{v\}} \rightarrow \mathrm{U}^{\{v\}}:\left\langle\mathrm{q}, \mathrm{x}^{v}, \mathrm{y}^{\mu}\right\rangle \mapsto \mathrm{u}^{v} \quad$ for $\varepsilon=0$,

ii) $\hat{\lambda}_{\mathrm{R}}:\{\mathrm{q}\} \times \mathrm{X}^{\{v\}} \times \mathrm{Y}^{\{v\}} \times \mathrm{Z}^{\{\mu\}} \rightarrow \mathrm{U}^{\{v\}}:\left\langle\mathrm{q}, \mathrm{x}^{v}, \mathrm{y}^{\mu}, \mathrm{z}^{\mu}\right\rangle \mapsto \mathrm{u}^{v} \quad$ for $\varepsilon=1$,

which can be formally (not actually) transformed to the form:

ad i) $\lambda_{\mathrm{R}}: \mathrm{X}^{\{v\}} \times \mathrm{Y}^{\{v\}} \rightarrow \mathrm{U}^{\{v\}}:\left\langle\mathrm{x}^{v}, \mathrm{y}^{\mu}\right\rangle \mapsto \mathrm{u}^{v}$ for $\varepsilon=0$

ad ii) $\lambda_{\mathrm{R}}: \mathrm{X}^{\{v\}} \times \mathrm{Y}^{\{v\}} \times \mathrm{Z}^{\{\mu\}} \rightarrow \mathrm{U}^{\{v\}}:\left\langle\mathrm{x}^{v}, \mathrm{y}^{\mu}, \mathrm{z}^{\mu}\right\rangle \mapsto \mathrm{u}^{v} \quad$ for $\varepsilon=1$.

Hence, the finite automaton model of a static logical object $\widehat{\mathrm{CA}}$ (which automatically performs virtual state transitions from $q$ to $q$ ) is the ordered triad

$$
\widehat{\mathrm{CA}}=\left\langle\mathrm{X} \times \mathrm{Y} \times \mathrm{Z}^{\varepsilon}, \mathrm{U}, \lambda_{\mathrm{R}}\right\rangle
$$

where $\lambda_{\mathrm{R}}$ is the Mealy output function:

i) $\lambda_{\mathrm{R}}: \mathrm{X}^{\{v\}_{\times}} \times \mathrm{Y}^{\{v\}} \rightarrow \mathrm{U}^{\{v\}}:\left\langle\mathrm{x}^{v}, \mathrm{y}^{\mu}\right\rangle \mapsto \mathrm{u}^{v}$ for $\varepsilon=0$,

ii) $\lambda_{\mathrm{R}}: \mathrm{X}^{\{v\}} \times \mathrm{Y}^{\{v\}} \times \mathrm{Z}^{\{\mu\}} \rightarrow \mathrm{U}^{\{v\}}:\left\langle\mathrm{x}^{v}, \mathrm{y}^{\mu}, \mathrm{z}^{\mu}\right\rangle \mapsto \mathrm{u}^{v} \quad$ for $\varepsilon=1$.

Let us construct an aggregation $\mathrm{O}(\widehat{\mathrm{CA}}, \mathrm{P})$ according to Fig. 3; since

$u^{v}=\lambda_{R}\left(x^{\nu}, y^{\mu}\right)=\lambda_{R}\left(x^{\nu}, \lambda\left(s^{\mu}\right)\right)$ for $\varepsilon=0$ and $u^{v}=\lambda_{R}\left(x^{\nu}, y^{\mu}, z^{\mu}\right)=\lambda_{R}\left(x^{v}, \lambda\left(s^{\mu}\right), z^{\mu}\right)$ for $\varepsilon=1$,

we will obtain:

i) $\delta\left(s^{\mu}, u^{v}, s^{\mu+1}\right)=\delta\left(s^{\mu}, \lambda_{R}\left(x^{\nu}, \lambda\left(s^{\mu}\right)\right), s^{\mu+1}\right), \quad$ spec. $\delta\left(s^{\mu}, u^{v}\right)=\delta\left(s^{\mu}, \lambda_{R}\left(x^{v}, \lambda\left(s^{\mu}\right)\right)\right)=s^{\mu+1}$

for $\varepsilon=0$,

ii) $\delta\left(s^{\mu}, u^{v}, z^{\mu}\right)=\delta\left(s^{\mu}, \lambda_{R}\left(x^{v}, \lambda\left(s^{\mu}\right), z^{\mu}\right), z^{\mu}\right)=\delta\left(s^{\mu}, \lambda_{R}\left(x^{v}, \lambda\left(s^{\mu}\right), z^{\mu}\right)\right)=s^{\mu+1}$ for $\varepsilon=1$.

Table 2: Transition table of the moving train from Example 2: a) scarce, b) satisfying

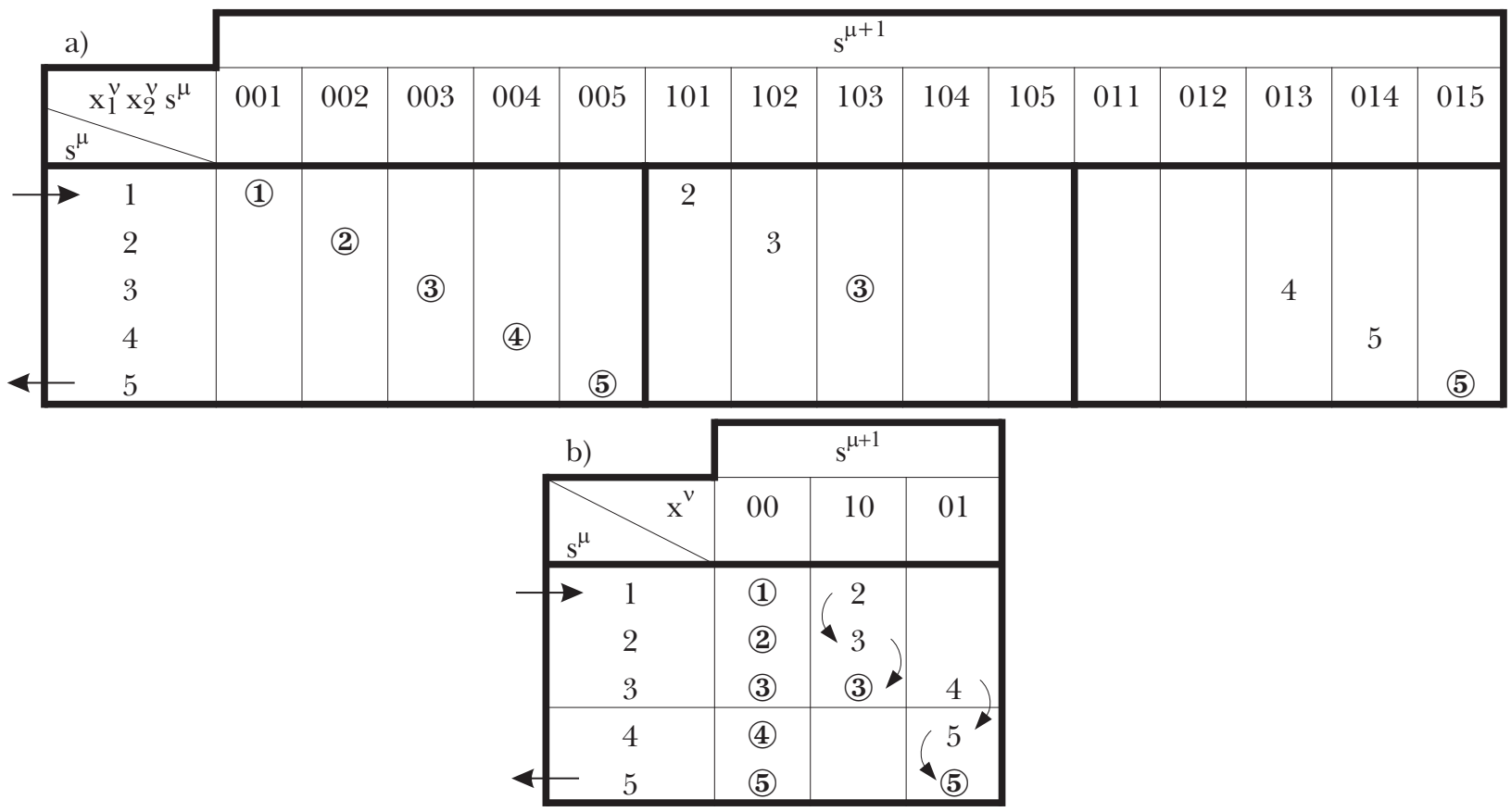




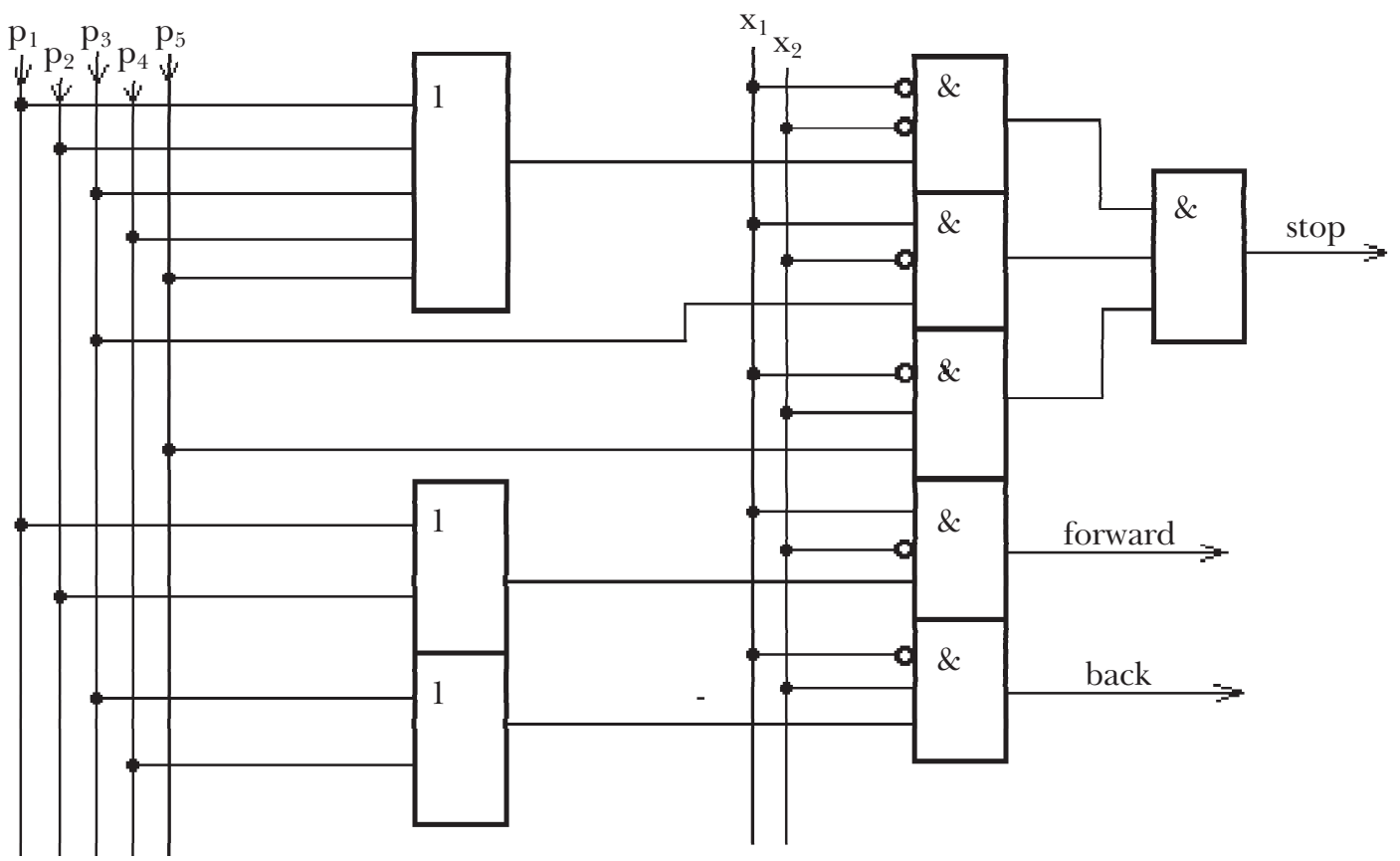

Fig. 4: Control automaton $\widehat{\mathrm{CA}}$ of the pseudotrain from Example 2

Then we can state that the finite-semi-automaton model of the logical entity $\boldsymbol{O}$ is the ordered triad

$$
\boldsymbol{O}=\left\langle\mathrm{X} \times \mathrm{Z}^{\varepsilon}, \mathrm{S}, \delta\right\rangle,
$$

where $\delta$ is a transition relation, spec. function, of the aggregation $\boldsymbol{O}(\widehat{\mathrm{CA}}, \mathrm{P})$ of dividends $\widehat{\mathrm{CA}}$ and $\mathrm{P}$ of object $\boldsymbol{O}$.

Example 2: Construct a $\widehat{C A}$ of the stepwise moving train from Example 1, in other words construct a logic object moving train. The transition table of such a moving train is presented in Tab. 2a). Since Tab. 2a) is scarce, we will use Tab. 2b) with arrows denoting the state transition of the object. The structural model of the dividend $\widehat{\mathrm{CA}}$ o of the train is $\mathrm{i} \mapsto 1$, if the state predicates of the pseudotrain $\mathrm{p}:\{\mathrm{i}\}_{i=1}^{5} \rightarrow\{0,1\}: \mathrm{i} \mapsto 0$ are introduced. Otherwise, the situation is as shown in Fig. 4.

In a justified way, a dynamic elementary logical object can be a matter of discussion and examination. Even when division of a logical entity is an intuitive concept (different from its decomposition), this also evidently holds: An elementary logical entity is another non - decomposable dynamic logical object. Let us, therefore, consider a delay $\vartheta=\left\langle D, \mathrm{~S}, \delta_{\mathrm{D}}\right\rangle$, be it deterministic or nondeterministic [5], where $D$ and $\mathrm{S}$ are the respective alphabets of the input and state delays, and $\delta_{\mathrm{D}}$ is the transition function $\mathrm{S}^{\{\mu\}} \times \mathrm{D}^{\{v\}} \rightarrow \rightarrow \mathrm{S}^{\{\mu+1\}}: \mathrm{D}^{v}=\mathrm{s}^{\mu+1}$. The equality $\mathrm{D}^{v}=\mathrm{s}^{\mu+1}$ states automatic production of state transition, and thus the delay is not only a dynamic elementary object, but even the only one elementary logical object.

Since the transition table of the logical object is scarce, whereas the transition table of the pseudoobject with added arrows depicting the automatism of performing state transitions is satisfying, the traditional finite - automaton pseudoobject models well also serve as finite - automaton models of dynamic entities provided that the stimuli from the objects only evoke state transitions, and the states produce them through the dividend $\widehat{C A}$ ( which is virtual in the case of the delay).

If $S=\varnothing$ is given in dividend $\mathrm{P}$ in the division of the object, we will obtain $\delta \subseteq \varnothing$ and $\lambda=\varnothing$ and the given entity is the static object $\widehat{\mathrm{CA}}$, because an object without a potentially $\mathrm{dy}$ namic dividend is sure to be static.

Note, in addition, that the connection of the level sensors or pulse sensors to the $\widehat{\mathrm{CA}}$ has to be ensured [5].

\section{Canonical decomposition}

Let us consider, without loss of generality, a dynamic prototype $\mathbf{O}$ traditionally modeled by a finite semiautomaton

$$
\mathbf{O}=\left\langle\mathrm{X} \times \mathrm{Z}^{\varepsilon}, \mathrm{S}, \delta\right\rangle
$$

where $\delta$ is a transition relation, spec. function, assigned in a traditional way:

i) $\delta \subseteq S^{\{\mu\}} \times X^{\{v\}} \times S^{\{\mu+1\}}: \delta\left(s^{\mu}, x^{v}, s^{\mu+1}\right)$,

$$
\text { spec. } \delta: S^{\{\mu\}} \times X^{\{v\}} \rightarrow S^{\{\mu+1\}}:\left\langle s^{\mu}, X^{v}\right\rangle \mapsto s^{\mu+1} \text {, for } \varepsilon=0
$$

ii) $\delta: S^{\{\mu\}} \times X^{\{v\}} \times Z^{\{\mu\}} \rightarrow S^{\{\mu+1\}}:\left\langle s^{\mu}, x^{v}, z^{\mu}\right\rangle \mapsto s^{\mu+1}$

for $\varepsilon=1$.

If we regard the state $s^{\mu}$, stimulus $x^{\nu}$, and failure $z^{\mu}$ as current, then $\mathrm{s}^{\mu+1}$ is:

i) $s^{\mu+1} \in \operatorname{proj}_{3}\left\{\delta\left(s^{\mu}, x^{\nu}, s^{\mu+1}\right)\right\}$,

spec. $s^{\mu+1}=\delta\left(s^{\mu}, x^{v}\right), \quad$ for $\varepsilon=0$,

ii) $s^{\mu+1}=\delta\left(s^{\mu}, x^{v}, z^{\mu}\right) \quad$ for $\varepsilon=1$ 


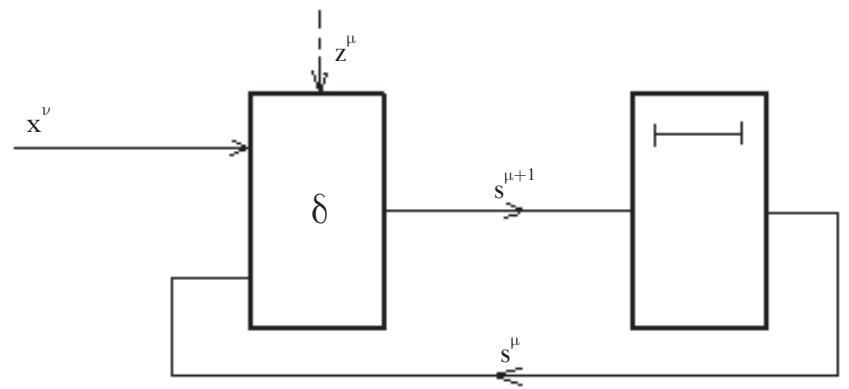

Fig. 5: Block interpretation of the transition relation, spec. function $\delta$ of object $\mathbf{O}$

a current prediction [6] of a state of the follower, and not the follower state itself, since the follower state cannot be currently documented. Then the composition $\mathbf{O}(\delta, \vartheta)$ from Fig. 5, where $\delta$ is a static, nondeterministic, spec. deterministic, logical object, can be regarded as a structural interpretation of the transition relation, spec. function $\delta$ of object $\mathbf{O}$, but not as its canonical decomposition, since the transition relation, spec. function does not regalement decomposition, and thus the canonical decomposition of a logical object. An interesting, and surprisingly common $[8,10,12]$ statement is that the static object $\delta$ according to the current $s^{\mu}, x^{\nu}$, or $z^{\mu}$ currently produces $\mathrm{s}^{\mu+1}$, which accepts nothing other than an anticipative delay?

Let us now leave the realm of prejudices, and since the relationship of covering the finite automata is not constructive [1], as it does not allow us to construct any automaton to the given automaton, let us, intuitively for the time being introduce an automaton, called a substitute, instead of the covering automaton. Let us consider the composition $\mathrm{O}_{\mathrm{N}}\left(\mathrm{I}, \mathrm{O}_{\mathrm{r}}\right)$ from Fig. 6, where $\mathrm{I}$ is the searched static deterministic exciting block of the given object $\mathrm{O}_{\mathrm{r}}-$ a substitute for pro-

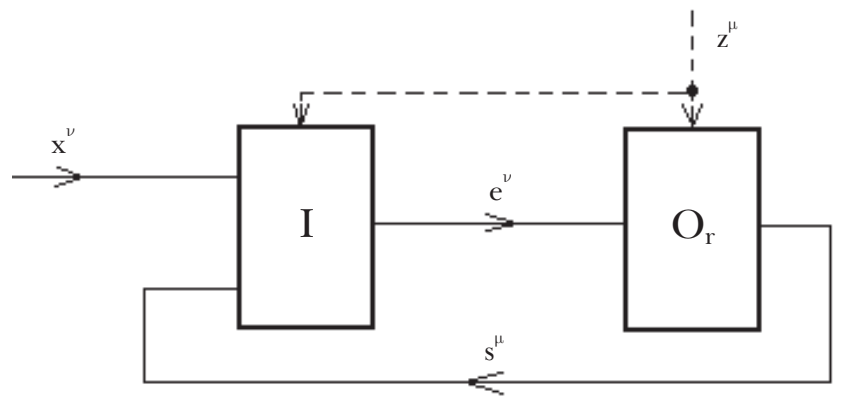

Fig. 6: Canonical composition $\mathrm{O}_{\mathrm{N}}\left(\mathrm{I}, \mathrm{O}_{\mathrm{r}}\right)$ of object $\mathbf{O}$

totype $\mathbf{O}$. Let the finite-semiautomaton model of $\mathrm{O}_{\mathrm{r}}$ be the ordered triad

$$
\mathrm{O}_{\mathrm{r}}=\left\langle\mathrm{E} \times \mathrm{Z}^{\varepsilon}, \mathrm{S}_{\mathrm{r}}, \delta_{\mathrm{r}}\right\rangle
$$

where $\mathrm{E}$ and $\mathrm{S}_{\mathrm{r}}$ are the respective exciting block alphabet and state alphabet, and $\delta_{\mathrm{r}}$ is the transition relation, spec. function:

i) $\delta_{\mathrm{r}} \subseteq \mathrm{S}_{\mathrm{r}}^{\{\mu\}} \times \mathrm{E}^{\{v\}} \times \mathrm{S}_{\mathrm{r}}^{\{\mu+1\}}: \delta_{\mathrm{r}}\left(\mathrm{s}_{\mathrm{r}}^{\mu}, \mathrm{e}^{v}, \mathrm{~s}_{\mathrm{r}}^{\mu+1}\right)$,

spec. $\delta_{\mathrm{r}}: \mathrm{S}_{\mathrm{r}}^{\{\mu\}} \times \mathrm{E}^{\{v\}} \rightarrow \mathrm{S}_{\mathrm{r}}^{\{\mu+1\}}:\left\langle\mathrm{s}_{\mathrm{r}}^{\mu}, \mathrm{e}^{v}\right\rangle \rightarrow \mathrm{s}_{\mathrm{r}}^{\mu+1}$ for $\varepsilon=0$

ii) $\delta_{\mathrm{r}}: \mathrm{S}_{\mathrm{r}}^{\{\mu\}} \times \mathrm{E}^{\{v\}} \times \mathrm{Z}^{\{\mu\}} \rightarrow \mathrm{S}_{\mathrm{r}}^{\{\mu+1\}}:\left\langle\mathrm{s}_{\mathrm{r}}^{\mu}, \mathrm{e}^{v}, \mathrm{z}^{\mu}\right\rangle \rightarrow \mathrm{s}_{\mathrm{r}}^{\mu+1}$,

for $\varepsilon=1$.

Let the finite-automaton model of the searched block I be the ordered triad

$$
\mathrm{I}=\left\langle\mathrm{X} \times \mathrm{S}_{\mathrm{r}} \times \mathrm{Z}^{\varepsilon}, \mathrm{E}, \lambda\right\rangle
$$

where $\lambda$ is the excitation function:
i) $\mathrm{X}^{\{\mu\}} \times \mathrm{S}_{\mathrm{r}}^{\{\mu\}} \rightarrow \mathrm{E}^{\{v\}}:\left\langle\mathrm{x}^{v}, \mathrm{~s}_{\mathrm{r}}^{\mu}\right\rangle \rightarrow \mathrm{e}^{v} \quad$ for $\varepsilon=0$
ii) $\mathrm{X}^{\{\mu\}} \times \mathrm{S}_{\mathrm{r}}^{\{\mu\}} \times \mathrm{Z}^{\{\mu\}} \rightarrow \mathrm{E}^{\{v\}}:\left\langle\mathrm{x}^{\nu}, \mathrm{s}_{\mathrm{r}}^{\mu}, \mathrm{z}^{\mu}\right\rangle \rightarrow \mathrm{e}^{v}$ for $\varepsilon=1$.

The finite-automaton model of the canonical decomposition $\mathrm{O}_{\mathrm{N}}\left(\mathrm{I}, \mathrm{O}_{\mathrm{r}}\right)$ is then the ordered triad

$$
\mathrm{O}_{\mathrm{N}}=\left\langle\mathrm{X}, \mathrm{S}_{\mathrm{r}}, \delta_{\mathrm{N}}\right\rangle
$$

where $\delta_{\mathrm{N}}$ is the transition relation, spec. function:

i) $\delta_{\mathrm{N}} \subseteq \mathrm{S}_{\mathrm{r}}^{\{\mu\}} \times \mathrm{X}^{\{v\}} \times \mathrm{S}_{\mathrm{r}}^{\{\mu+1\}}: \delta_{\mathrm{N}}\left(\mathrm{s}_{\mathrm{r}}^{\mu}, \mathrm{x}^{\nu}, \mathrm{s}_{\mathrm{r}}^{\mu+1}\right)$, spec. $\delta_{\mathrm{N}}: \mathrm{S}_{\mathrm{r}}^{\{\mu\}} \times \mathrm{X}^{\{v\}} \rightarrow \mathrm{S}_{\mathrm{r}}^{\{\mu+1\}}:\left\langle\mathrm{s}_{\mathrm{r}}^{\mu}, \mathrm{x}^{v}\right\rangle \rightarrow \mathrm{s}_{\mathrm{r}}^{\mu+1}$ for $\varepsilon=0$,

ii) $\delta_{\mathrm{r}}: \mathrm{S}_{\mathrm{r}}^{\{\mu\}} \times \mathrm{X}^{\{v\}} \times \mathrm{Z}^{\{\mu\}} \rightarrow \mathrm{S}_{\mathrm{r}}^{\{\mu+1\}}:\left\langle\mathrm{s}_{\mathrm{r}}^{\mu}, \mathrm{x}^{v}, \mathrm{z}^{\mu}\right\rangle \rightarrow \mathrm{s}_{\mathrm{r}}^{\mu+1}$ for $\varepsilon=1$.

The composition $\mathrm{O}_{\mathrm{N}}\left(\mathrm{I}, \mathrm{O}_{\mathrm{r}}\right)$ from Fig. 6 [5] is regarded as a canonical decomposition of prototype $\mathbf{O}$, if there exists the monomorphism $\mathbf{O}$ to $\mathrm{O}_{\mathrm{N}}$, i.e., if with the given coding state $\mathrm{K}: \mathrm{S} \rightarrow \mathrm{S}_{\mathrm{r}}: \mathrm{s} \mapsto \mathrm{s}_{\mathrm{r}}$ the following relation holds:

i) $\delta\left(s^{\mu}, x^{\nu}, s^{\mu+1}\right) \Rightarrow \delta\left(K\left(s^{\mu}\right), x^{\nu}, K\left(s^{\mu+1}\right)\right)$, spec. $K\left(\delta\left(s^{\mu}, x^{\nu}\right)\right)=\delta_{N}\left(K\left(s^{\mu}\right), x^{\nu}\right)$, for $\varepsilon=0$,

ii) $K\left(\delta\left(s^{\mu}, x^{\nu}, z^{\mu}\right)\right)=\delta_{N}\left(K\left(s^{\mu}\right), x^{\nu}, z^{\mu}\right)$ for $\varepsilon=1$.

Since

i) $\operatorname{proj}_{3}\left\{\delta_{\mathrm{N}}\left(\mathrm{K}\left(\mathrm{s}^{\mu}\right), \mathrm{x}^{v}, \mathrm{~K}\left(\mathrm{~s}^{\mu+1}\right)\right)\right\}=\operatorname{proj}_{3}\left\{\delta_{\mathrm{r}}\left(\mathrm{K}\left(\mathrm{s}^{\mu}\right), \mathrm{e}^{\nu}, \mathrm{K}\left(\mathrm{s}^{\mu+1}\right)\right)\right\}=$

$=\operatorname{proj}_{3}\left\{\delta_{\mathrm{r}}\left(\mathrm{K}\left(\mathrm{s}^{\mu}\right), \lambda\left(\mathrm{x}^{\nu}, \mathrm{K}\left(\mathrm{s}^{\mu}\right)\right), \mathrm{K}\left(\mathrm{s}^{\mu+1}\right)\right)\right\}$,

spec. $\delta_{N}\left(K\left(s^{\mu}\right), x^{v}\right)=\delta_{r}\left(K\left(s^{\mu}\right), e^{v}\right)=\delta_{r}\left(K\left(s^{\mu}\right), \lambda\left(x^{v}, K\left(s^{\mu}\right)\right)\right)$, for $\varepsilon=0$,

ii) $\delta_{N}\left(K\left(s^{\mu}\right), x^{\nu}, z^{\mu}\right)=\delta_{r}\left(K\left(s^{\mu}\right), e^{\nu}, z^{\mu}\right)=\delta_{r}\left(K\left(s^{\mu}\right), \lambda\left(x^{\nu}, K\left(s^{\mu}\right), z^{\mu}\right), z^{\mu}\right)$ for $\varepsilon=1$ 
the searched excitation $\mathrm{e}^{v}$ can be found, as

i) $\delta_{N}\left(s^{\mu}, x^{v}, s^{\mu+1}\right) \Rightarrow \delta\left(K\left(s^{\mu}\right), \lambda\left(x^{v}, K\left(s^{\mu}\right)\right), K\left(s^{\mu+1}\right)\right)$,
spec. $K\left(\delta_{N}\left(s^{\mu}, x^{v}\right)\right)=\delta_{r}\left(K\left(s^{\mu}\right), \lambda\left(x^{v}, K\left(s^{\mu}\right)\right)\right)$, for $\varepsilon=0$,
ii) $K\left(\delta_{N}\left(s^{\mu}, x^{v}, z^{\mu}\right)\right)=\delta_{r}\left(K\left(s^{\mu}\right), \lambda\left(x^{v}, K\left(s^{\mu}\right), z^{\mu}\right), z^{\mu}\right)$ for $\varepsilon=1$.

Note that the substitute $\mathrm{O}_{\mathrm{r}}$, intuitive at first, is exactly defined by the monomorphism $\mathbf{O}$ into $\mathrm{O}_{\mathrm{N}}$.

The universal substitute $\mathrm{O}_{\mathrm{r}}$ of prototype $\mathbf{O}$ is an entity such that:

- $\left|S_{r}\right| \geq|S|$,

- it can have every possible state transition.

Example 3: Verify whether the object according to Tab. 3a) is the proper substitute of the simulated train from Tab. 1a) by constructing its excitation (Tab. 3b)) at state coding $\mathrm{K}:\{i\}_{i=1}^{5} \rightarrow\{j\}_{j=1}^{\mathrm{VI}}: 1 \mapsto \mathrm{I}, 2 \mapsto \mathrm{III}, 3 \mapsto \mathrm{IV}, 4 \mapsto \mathrm{V}, 5 \mapsto \mathrm{VI}$

Table 3: a) Transition table of the substitute object of the train, b) Excitation table of the substitute

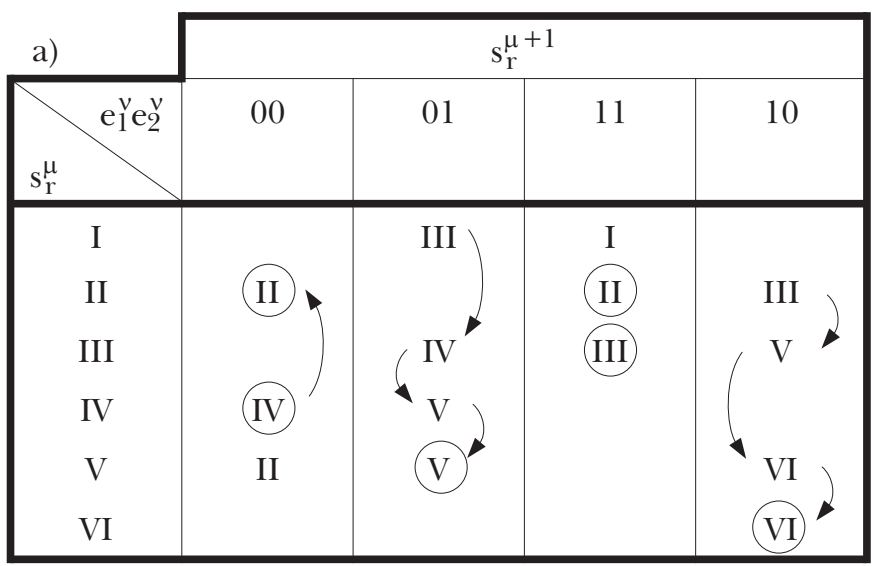

\begin{tabular}{|c|c|c|c|c|c|c|c|}
\cline { 3 - 7 } \multicolumn{2}{c|}{ b) } & \multicolumn{3}{c|}{$\mathrm{s}_{\mathrm{r}}^{\mu+1}$} & \multicolumn{3}{|c|}{$\mathrm{e}_{1}^{\nu} \mathrm{e}_{2}^{\nu}$} \\
\hline $\mathrm{s}^{\mu}$ & $\mu_{1}^{\nu} \mu_{2}^{\nu}$ & 00 & 01 & 10 & 01 & 10 & 01 \\
\hline 1 & $\mathrm{I}$ & $\mathrm{I}$ & $\mathrm{III}$ & & 11 & 01 & \\
& $\mathrm{II}$ & & & & & & \\
2 & $\mathrm{III}$ & $\mathrm{III}$ & $\mathrm{IV}$ & & 11 & 01 & \\
3 & $\mathrm{IV}$ & $\mathrm{IV}$ & $\mathrm{IV}$ & $\mathrm{V}$ & 00 & 00 & 01 \\
4 & $\mathrm{~V}$ & $\mathrm{~V}$ & & $\mathrm{VI}$ & 01 & & 10 \\
5 & $\mathrm{VI}$ & $\mathrm{VI}$ & & $\mathrm{VI}$ & 10 & & 10 \\
\hline
\end{tabular}

The structural interpretation of the transition relation, spec. function, $\delta$ of object $\mathbf{O}$ can be considered as a canonical decomposition of entity $\mathbf{O}$ if instead of the current prediction $\mathrm{s}^{\mu+1}$ though an actual, as is only correct, excitation $\mathrm{D}^{v}$ of the delay can be introduced, since $\mathrm{D}^{v}=\mathrm{s}^{\mu+1}$. Note that if there did not exist at least one dynamic elementary logic object, a structural model of a dynamic logic entity could not be constructed. Let us have in mind that a universal and, in a limited way, simple binary dynamic substitute of the binary dynamic object considered is a parallel register (the simplest possible composition) of binary dynamic memory modules. In this case, any structural model of an arbitrary binary memory module, designed either intuitively or exactly (by canonical decomposition), is strongly dependent on the binary dynamic elementary substitute - on a binary delay. Therefore the structural models of a computational, pseudoobject designed by canonical decomposition with substitutes, which are always logic objects, are also logic objects, which have not yet been taken into account, and the process of controlling a logic object, as can be seen from paragraph 2 , does not make sense.

There is also an obvious extremely large difference between the division of $\widehat{\mathrm{CA}}$ an object and its ca- $\widehat{\mathrm{CA}}$ nonical decomposition; even though and I are static object, controls the given pseudoobject $\mathrm{B}$, whereas I "forces" the substitute $\mathrm{O}_{\mathrm{r}}$ to mimic by its transition sequences the transition sequences on the given prototype $\mathbf{O}$.

\section{State of the object}

Let us consider an extended finite-automaton model

$$
\mathrm{O}=\left\langle\mathrm{X} \times \mathrm{Z}^{\varepsilon}, \mathrm{S}, \mathrm{Y}, \delta, \lambda\right\rangle
$$

of a logic entity $\mathrm{O}$, where $\mathrm{Y}$ is the output alphabet and $\lambda$ can be either a Mealy output relation, spec. function:

i) $\lambda \subseteq S^{\{\mu\}} \times X^{\{v\}} \times Y^{\{\mu\}}: \lambda\left(s^{\mu}, x^{v}, y^{\mu}\right)$,

$$
\text { spec. } \lambda: S^{\{\mu\}} \times X^{\{v\}} \rightarrow Y^{\{\mu\}}:\left\langle s^{\mu}, x^{\nu}\right\rangle \mapsto y^{\mu} \text {, for } \varepsilon=0 \text {, }
$$

ii) $\lambda: S^{\{\mu\}} \times \mathrm{X}^{\{v\}} \times \mathrm{Z}^{\{\mu\}} \rightarrow \mathrm{Y}^{\{\mu\}}:\left\langle\mathrm{s}^{\mu}, \mathrm{x}^{\nu}, \mathrm{z}^{\mu}\right\rangle \mapsto \mathrm{y}^{\mu}$, for $\varepsilon=1$,

or a Moore output function

$$
\lambda: S^{\{\mu\}} \rightarrow Y^{\{\mu\}}: \mathrm{s}^{\mu} \mapsto \mathrm{y}^{\mu} .
$$

The finite-automaton model of a logic entity is evidently introduced:

- independent of the content of the "state of the object" concept,

- in such a way that the state of the entity both parameterizes [9], [13] an arbitrary input/output dyad $\left\langle x^{\nu}, y^{\mu}\right\rangle$, and transfers each state transition; though there are an unlimited number of ways of parametrizing/transferring, all of them are substantially equivalent.

In $[7,11]$ the state of the object is understood as the entire input history of the entity being recorded in a cumulative way (without deletion) and stored in the "memory", i.e., in the 
delay of block interpretation of the transition relation, spec. function, $\delta$ of object $\mathrm{O}$. But if it is

$$
\begin{aligned}
& \text { i) } \mathrm{s}^{\mu+1} \in \operatorname{proj} 3\left\{\delta\left(\mathrm{s}_{i}^{\mu}, \mathrm{x}^{\nu}, \mathrm{s}^{\mu+1}\right)\right\} \cap \operatorname{proj3}\left\{\delta\left(\mathrm{s}_{j}^{\mu}, \mathrm{x}^{\nu}, \mathrm{s}^{\mu+1}\right)\right\}, \\
& \text { spec. } \mathrm{s}^{\mu+1}=\delta\left(\mathrm{s}_{i}^{\mu}, \mathrm{x}^{\nu}\right)=\delta\left(\mathrm{s}_{j}^{\mu}, \mathrm{x}^{\nu}\right) \text {, for } \varepsilon=0, \\
& \text { ii) } \mathrm{s}^{\mu+1}=\delta\left(\mathrm{s}_{i}^{\mu}, \mathrm{x}^{\nu}, \mathrm{z}^{\mu}\right)=\delta\left(\mathrm{s}_{j}^{\mu}, \mathrm{x}^{\nu}, \mathrm{z}^{\mu}\right) \text {, for } \varepsilon=1,
\end{aligned}
$$

how can the input history $\mathrm{s}_{i}^{\mu} \neq \mathrm{s}_{j}^{\mu}$, i.e., for different input histories cumulatively included in $\mathrm{s}_{i}^{\mu}$ and in $\mathrm{s}_{j}^{\mu}$, the input history, which originated by connecting $\mathrm{x}^{v}$, or $\mathrm{x}^{v} \mathrm{z}^{\mu}$ to $\mathrm{s}_{i}^{\mu}$ or to $\mathrm{s}_{j}^{\mu}$, respectively, be exactly identical with $s^{\mu+1}$ ? The memory of the entity so does not cumulatively record and does not store the input history of an entity, and therefore the conception of the object according to Fig. 5 is not tenable, though it may appear sufficient to put $s^{\mu+1}=D^{v}$, where $D^{v}$ is the excitation of the delay.

\section{Instead of a conclusion}

The authors, though having found that a state not only parameterizes the input/output pair but also transfers the state transition, which is relevant, regret to say that by rejecting the current conception of the state they have made the content of the concept "state" a little unclear.

They also believe that objections such as:

- I am not convinced that logic control is combinational,

- the requirement for $\widehat{\mathrm{CA}}:|\mathrm{Q}|=1$ is too high,

- logic control is not so simple as some would wrongly think,

- in canonical decomposition both components are to be determined,

- in canonical decomposition the state can be measured,

will be found insubstantial by the reader.

\section{References}

[1] Baranov, S. I.: Sintez mikroprogrammnych avtomatov. Energija, Leningrad, 1979
[2] Bokr, J. at al: Logické ř́zení technologických procesů. SNTL, Praha, 1986

[3] Bokr, J., Jáneš, V.: Logic Control and Canonical Decomposition. In: Proceedings of UWB, Vol. 1, pp. 33-40, Plzeň, 1997

[4] Bokr, J.: Upravlenie logičeskim objektom i kanoničeskaja dekompozicija. AVT, No. 6/1999, pp. 12-23

[5] Bokr, J., Jáneš, V.: Logické systémy. Vydavatelství ČVUT, Praha, 1999

[6] Bokr, J., Svatek, J.: Základy logiky a argumentace. A. Čeněk, Plzeň, 2000

[7] Brunovský, P., Černý, J.: Základy matematickej teórie systémov. Veda, Bratislava, 1980

[8] Frištacký, N. at al: Logické systémy. ALFA-SNTL, Bratislava-Praha, 1986

[9] Kalman, R. E., Falb, P. L., Arbib, M.: Topics in Mathematical System Theory. Mc Graw - Hill Book Co., 1969

[10] Katz, H. R.: Contemporary Logic Design. The Benjamin Cummings Publishing Co., Inc., 1994

[11] Minsky, M. L.: Finite and Infinite Machines. Russian translation, Mir Moskva, 1971

[12] Perrin, J. P., Denouette, M., Daclin, E.: Systemes logiques. Dunod, Paris, 1967

[13] Zadeh, L. A.: The Concept of State in System Theory. In: Views on General System Theory, edited by Mesarovič, M. D., J. Wiley \& Sons, Inc., New York-London-Sydney, 1964

Doc. Ing. Josef Bokr, CSc.

Department of Information and Computer Science

University of West Bohemia

Faculty of applied Sciences

Universitní 22

30614 Pilsen, Czech Republic

Doc. Ing. Vlastimil Jáneš, CSc.

Department of Computer Science and Engineering

Czech Technical University in Prague

Faculty of Electrical Engineering

Karlovo nám. 13, 12125 Praha 2, Czech Republic 